"skill utilisation" and "physician-nurse relation" determined workengagement.

Conclusions A slightly higher number of Belgian doctors suffered from burnout compared to previous studies, yet also showed work-engagement. Further analysis on determinants will be conducted as a base for preventive measures and actions to improve work-engagement.

\section{NIGHT SHIFT WORK AND PROLACTIN IN NURSES AND MIDWIVES - PRELIMINARY RESULTS}

A B Bukowska, Peplonska, Sobala. Nofer Institute of Occupational Medicine, Lodz, Poland

\subsection{6/oemed-2013-101717.374}

Objectives Night shift work and exposure to light at night suppress synthesis of melatonin and disrupt circadian rhythm. The pattern of secretion of many hormones, including prolactin, is dependent on circadian rhythm and prolactin has been found to play a role in breast cancer aetiology. So far, the data on the link between night shift work and prolactin are sparse. The aim of our study was to evaluate the association between rotating night shift work and prolactin concentration in nurses and midwives.

Methods The cross-sectional study included 347 nurses and midwives currently working on rotating night shifts and 359 nurses and midwives working during the day. The prolactin concentration was measured in the morning blood samples using the ECLIA method. The associations were estimated by multiple linear regression models adjusted for: age, number of full-term births, current oral contraceptives use and time of blood collection. A potential modification by the menopausal status was analysed.

Results No difference in the prolactin concentration was found between the women currently working on night shifts and the day nurses (geometric means (GM): $195.3 \mu \mathrm{U} / \mathrm{ml}$ vs. $194.7 \mu \mathrm{U} /$ $\mathrm{ml} ; \mathrm{p}=0.948)$. In women with 2 or more consecutive night shifts within one week before the blood collection, we observed a statistically significant increase of the prolactin concentration compared to the women who had only one night shift throughout that week $(250.2 \mu \mathrm{U} / \mathrm{ml}$ vs. $216.9 \mu \mathrm{U} / \mathrm{ml} ; \mathrm{p}=0.033)$. None of the examined associations were modified by the menopausal status.

Conclusions The preliminary results of our study suggest that night shift work might modify prolactin secretion.

\section{THE PREVALENCE OF LATEX SENSITISATION AND ALLERGY AND ASSOCIATED RISK FACTORS AMONG HEALTHCARE WORKERS USING HYPOALLERGENIC LATEX GLOVES SOUTH AFRICA}

S M Phaswana, Naidoo. University of KwaZulu Natal, Durban, South Africa

\subsection{6/oemed-2013-101717.375}

Objectives The present study describes latex sensitisation and allergy prevalences and associated factors among healthcare workers using hypoallergenic latex gloves at King Edward VIII Hospital in KwaZulu-Natal South Africa.

Methods A cross sectional survey of randomly selected healthcare workers $(\mathrm{n}=501)$, (337 exposed and 164 unexposed) at King Edward VIII Hospital, South Africa was conducted between 2011 and 2012. Healthcare worker data was collected using a questionnaire and skin prick tests using the Stallergenes kit. Chisquare and Kruskal-Wallis test were used for significance testing on bivariate analysis. Binary logistic regression was used to test associations between dependent and independent outcomes on multivariate analysis.

Results Prevalence of latex sensitisation and allergy observed among exposed workers (7.1\% and 5.9\%) and unexposed workers $(3.1 \%$ and $1.8 \%)$. Work related allergy symptoms were significantly higher in exposed workers (40.9\%, p $<0.05)$. Duration of employment was protective against latex allergy (OR: 0.9 ; 95\% CI: 0.8-0.9). The risk of latex sensitisation (OR: 4.2; 95\% CI: 1.2-14.1) and allergy (OR: 5.1; 95\% CI: $1.2-$ 21.2) increased with exclusive use of powder-free latex gloves. Dose -response relationship was observed for powdered latex gloves (OR: 1.1; 95\% CI: 1.0-1.2). Atopy (OR: 1.5; 95\% CI: $0.7-3.3$ and $1.4 ; 95 \% \mathrm{CI}: 0.6-3.2$ ) and fruit allergy (OR: 2.3; 95\% CI: $0.8-6.7$ and 3.1; 95\% CI: 1.1-9.2) also increased the risk of latex sensitisation and allergy, respectively. The observed relation between atopy and latex sensitisation and allergy was not significant.

Conclusion This study adds to previous findings that healthcare workers exposed to hypoallergenic latex gloves are at risk for developing latex sensitisation highlighting its importance as an occupational hazard in healthcare.

\section{THE PREVALENCE, CIRCUMSTANCES AND REPORTING OF PATIENT/VISITOR-ON-WORKER (TYPE II) VIOLENCE IN 6 U. S. HOSPITALS}

${ }^{1}$ L P Pompeii, ${ }^{2}$ Schoenfisch, ${ }^{2}$ Dement, ${ }^{1}$ Jones, ${ }^{2}$ Hester, ${ }^{3}$ Smith. ${ }^{1}$ The University of Texas, Houston, United States of America; '2Duke University Medical Center, Durham, NC, United States of America; ${ }^{3}$ St. Luke's Episcopal Hospital, Houston, Texas, United States of America

\subsection{6/oemed-2013-101717.376}

Objectives We aimed to establish baseline measures of type II violence (patient/visitor-on-worker) and event reporting practices among workers in $6 \mathrm{U}$. S. hospitals. Findings from this survey will inform the development and implementation of a hospital violence surveillance system.

Methods An anonymous cross-sectional survey was administered to workers to examine their career and 12-month prevalence of being victims of workplace violence perpetrated by patients and/ or visitors, as well as details about events that workers deemed the most serious.

Results Of the 5,312 hospital workers who responded, 49.4\% and $38.9 \%$ reported a career and 12-month prevalence of type II violence, respectively. The total number of events in the prior 12 months included 1,100 physical assaults, 2,206 physical threats, and 5,676 verbal abuse events. More than half of the events were reported by nursing staff $(36.7 \%)$ and patient care technologists $(14.8 \%)$. Other workgroups not typically considered to be at risk also reported events including those in pharmacy, social work, food service, housekeeping, and patient financial services. Of the 2,098 most serious events in the prior year, perpetrators were more likely to be patients $(76.1 \%)$ than visitors $(23.9 \%)$. Factors that workers attributed to these events included patients' behavioural issues, being disoriented, being drunk, and visitors being unhappy with patient's care and long wait-times. A large proportion (75.0\%) of workers indicated they reported the event; however, only a small proportion of those $(26.2 \%)$ did so through a formal system (e.g., first report 
of injury), while most reported verbally (58.9\%) to coworkers/ managers or documented in the patient's chart (14.9\%).

Conclusion While the prevalence and number of violent events was high, the reporting of events by workers into a formal system was low. Reporting systems developed specifically for capturing type II violent events are needed for purposes of informing and evaluating targeted workplace violence prevention strategies.

\section{Session: 30. Lymphoma and leukemia}

\section{OCCUPATION AND RISK OF LYMPHOID AND MYELOID LEUKEMIA IN THE EUROPEAN PROSPECTIVE INVESTIGATION INTO CANCER AND NUTRITION (EPIC)}

${ }^{1} \mathrm{~F}$ Saberi Hosnijeh, ${ }^{1}$ Christopher, ${ }^{2}$ Peeters, ${ }^{3}$ Romieu, ${ }^{4}$ Xun, ${ }^{4}$ Riboli, ${ }^{5}$ Raaschou-Nielsen, ${ }^{5}$ Tjønneland, ${ }^{6}$ Becker, ${ }^{7}$ Nieters, ${ }^{8}$ Trichopoulou, ${ }^{8}$ Bamia, ${ }^{9}$ Orfanos, ${ }^{10}$ Oddone, ${ }^{11}$ LujánBarroso, ${ }^{12}$ Dorronsoro, ${ }^{13}$ Navarro, ${ }^{14}$ Barricarte, ${ }^{15}$ Molina-Montes, ${ }^{16}$ Wareham, ${ }^{4}$ Vineis, ${ }^{1}$ Vermeulen. 'Utrecht University, Utrecht, The Netherlands; ${ }^{2}$ University Medical Center Utrecht, Utrecht, The Netherlands; ${ }^{3}$ 4International Agency for Research on Cancer, Lyon, France; ${ }^{4}$ Imperial College, London, United Kingdom; ${ }^{5}$ Danish Cancer Society Research Center, Copenhagen, Denmark; ${ }^{6} D K F Z$, Heidelberg, Germany; ${ }^{7}$ University of Freiburg, Freiburg, Germany; ${ }^{8}$ University of Athens Medical School, Athens, Greece; ${ }^{9}$ Hellenic Health Foundation, Athens, Greece; ${ }^{10}$ Fondazione IRCCS Istituto Nazionale dei Tumori, Milan, Italy; ${ }^{11}$ Catalan Institute of Oncology (ICO), Barcelona, Spain; ${ }^{12}$ Basque Regional Health Department and Biodonostia, Ciberesp, Spain; ${ }^{13}$ Murcia Regional Health Authority, Murica, Spain; ${ }^{14}$ Navarre Public Health Institute, Pamplona, Spain; ${ }^{15}$ Andalusian School of Public Health, Granada, Spain; ${ }^{16}$ Medical Research Council, Epidemiology Unit, Cambridge, United Kingdom

\subsection{6/oemed-2013-101717.377}

Objectives Established risk factors of leukemia do not explain the majority of leukemia cases. Previous studies have suggested the importance of occupation and related exposures in leukemogenesis. We evaluated possible associations between job title and selected hazardous agents and leukemia in the European Prospective Investigation into Cancer and Nutrition.

Methods The mean follow-up time for 241,465 subjects was 11.20 years (SD: 2.42 years). During the follow-up period, 477 incident cases of myeloid and lymphoid leukemia occurred. Data on 52 occupations considered a priori to be at high risk for developing cancer were collected through standardized questionnaires. Occupational exposures were estimated by linking the reported occupations to a Job exposure matrix. Cox proportional hazard models were used to explore the association between occupation and related exposures and risk of leukemia. Results Risk of lymphoid leukemia significantly increased for working in chemical laboratories $(\mathrm{HR}=8.35,95 \% \mathrm{CI}=1.58-$ 44.24), while the risk of myeloid leukemia increased for working in the shoes or other leather goods industry (HR $=2.54,95 \%$ $\mathrm{CI}=1.28-5.06)$. Exposure specific analyses showed a non-significant increased risk of myeloid leukemias for exposure to benzene $(\mathrm{HR}=1.15,95 \% \mathrm{CI}=0.75-1.40 ; \mathrm{HR}=1.60,95 \% \mathrm{CI}$ $=0.95-2.69$ for the low and high exposure categories respectively). This association was present both for acute and chronic myeloid leukemia at high exposure levels. However, numbers were too small to reach statistical significance.

Conclusion Our findings suggest a possible role of occupational exposures in development of both lymphoid and myeloid leukemia. Exposure to benzene seemed to be associated with both acute and chronic myeloid leukemia.

\section{OCCUPATION AND RISK OF NON-HODGKIN LYMPHOMA (NHL) AND SUBTYPES: A POOLED ANALYSIS FROM THE INTERLYMPH CONSORTIUM}

${ }^{1} \mathrm{~A}$ M 't Mannetie, ${ }^{2}$ De Roos, ${ }^{3}$ Boffetta, ${ }^{4} \mathrm{Cocco},{ }^{5}$ Benke, ${ }^{6} \mathrm{Blair},{ }^{7}$ Brennan, ${ }^{8} \mathrm{Chiu},{ }^{9} \mathrm{Clavel}$, ${ }^{10}$ De Sanjose, ${ }^{11}$ Hartge, ${ }^{12}$ Holly, ${ }^{13}$ Roman, ${ }^{14}$ Seniori Costantini, ${ }^{15}$ Spinelli, ${ }^{16}$ Zheng, ${ }^{17}$ Kricker. ${ }^{1}$ Massey University, Wellington, New Zealand; ${ }^{2}$ Fred Hutchinson Cancer Research Center, Seattle, United States of America; ${ }^{3}$ IPRI, Lyon, France; ${ }^{4}$ University of Cagliari, Cagliari, Italy; ${ }^{5}$ University of Melbourne, Melbourne, Australia; ${ }^{6}$ National Cancer Institute, Washington, United States of America; ${ }^{7}$ IARC, Lyon, France; ${ }^{8}$ University of Chicago, Chicago, United States of America; ${ }^{9}$ Inserm, Villejuif, France; ${ }^{10}$ Catalan Institute of Oncology, Barcelona, Spain; ${ }^{11} \mathrm{NCl}$, Washington, United States of America; ${ }^{12}$ University of California, San Francisco, United States of America; ${ }^{13}$ University of York, York, United Kingdom; ${ }^{14}$ Center for Study and Prevention of Cancer, Florence, Italy; ${ }^{15} B C$ Cancer Research Center, Vancouver, Canada; ${ }^{16}$ Yale school of Public Health, New Haven, United States of America; ${ }^{17}$ University of Sydney, Sydney, Australia

\subsection{6/oemed-2013-101717.378}

Objectives A range of occupations have been associated inconsistently with an elevated NHL risk. In this large, pooled study, we investigate the relationship between occupation and NHL and NHL subtypes.

Methods This pooled study of 10 NHL case-control studies participating in the InterLymph consortium, included 10,046 cases uniformly classified by subtype and 12,025 controls. Occupational histories were classified according to the ISCO 1968 classification, and occupations previously associated with increases in hematologic cancer risk were grouped into 26 a priori high risk occupational groups. Odds ratios, adjusting for centre, age and sex were determined for the a priori groups as well as all ISCO occupational codes including a minimum of 10 cases. Analyses were repeated by sex and for the subtypes diffuse large B-cell lymphoma (DLBCL; $\mathrm{n}=3,061$ ), follicular lymphoma (FL; $\mathrm{n}=2,140$ ), chronic lymphocytic leukemia/small lymphocytic lymphoma (CLL/SLL; $\mathrm{n}=1,014)$ and T-cell lymphoma $(\mathrm{n}=632)$.

Results DLBCL risk was elevated for textile workers (OR: 1.19; 95\%CI: 1.01-1.41); field crop and vegetable farm workers $(1.50 ; 1.15-1.97)$; charworkers, cleaners and related workers $(1.27 ; 1.03-1.58)$ and hairdressers $(1.47 ; 1.08-2.00)$. FL risk was elevated for unspecified labourers $(1.28 ; 1.06-1.55)$ and spray painters $(2.67 ; 1.36-5.25)$. CLL/SLL risk was elevated for women's hairdressers $(2.69 ; 1.43-5.05)$; general farm workers (1.44; $1.13-1.84)$; pre-primary education teachers $(2.00 ; 1.04$ $3.87)$ and printing pressmen $(6.52 ; 2.79-15.2)$. T-cell lymphoma risk was elevated for textile workers $(1.60 ; 1.18-2.17)$; wood workers $(1.54 ; 1.04-2.27)$ and painters $(1.80 ; 1.14-2.84)$. ORs differed significantly among subtypes for hairdressers, textile workers and printing pressmen.

Conclusions This pooled analysis supports a role for farming, textile, and hairdressing related exposures in the development of NHL. Occupations with potential exposure to solvents, metals, wood dust, infectious agents and mineral dust were also positively associated with NHL. For all four studied NHL subtypes occupational risk factors play a role, with notable differences in risk occupations across subtypes.

\section{OCCUPATIONAL EXPOSURE TO TRICHLOROETHYLENE AND RISK OF NON-HODGKIN LYMPHOMA AND ITS MAJOR SUBTYPES: A POOLED INTERLYMPH ANALYSIS}

${ }^{1} \mathrm{P}$ C Cocco, ${ }^{2}$ Vermeulen, ${ }^{1}$ Flore, ${ }^{1}$ Nonne, ${ }^{1}$ Campagna, ${ }^{3}$ Purdue, ${ }^{3}$ Blair, ${ }^{4}$ Monnereau, ${ }^{5}$ Orsi, ${ }^{6}$ Becker, ${ }^{7}$ De Sanjosé, ${ }^{8}$ Foretova, ${ }^{9}$ Staines, ${ }^{10}$ Maynadié, ${ }^{11}$ Nieters, ${ }^{12}$ Miligi, ${ }^{13}$ 't Mannetje, ${ }^{14}$ Brennan, ${ }^{3}$ Lan, ${ }^{3}$ Rothman. ${ }^{1}$ University of Cagliari, Monserrato, Italy; ${ }^{2}$ Institute for Risk Assessment Sciences, Utrecht University, Utrecht, The Netherlands; ${ }^{3}$ Division of Cancer Epidemiology and Genetics, National Cancer Institute, NIH, Bethesda, Maryland, United States of America; ${ }^{4}$ Centre régional de lutte contre le cancer, Institut Bergonié, Bordeaux, 